\title{
Breather Induction by Modulational Instability in Binary Metamaterials
}

\author{
N. LAZARIDES ${ }^{a, *}$, M.I. MOLInA ${ }^{b}$ AND G.P. Tsironis ${ }^{a}$ \\ ${ }^{a}$ Department of Physics, University of Crete, and FORTH, P.O. Box 2208, 71003 Heraklion, Greece \\ ${ }^{b}$ Departamento de Fisica, Facultad de Ciencias, Universidad de Chile, Casilla 653, Santiago, Chile \\ It is demonstrated numerically that nonlinear localized excitations in the form of dissipative discrete \\ breathers can be induced spontaneously through the mechanism of modulational instability in nonlinear magnetic \\ metamaterials comprised of binary arrays of split-ring resonators. Specifically, a variety of high amplitude \\ dissipative breathers can be produced by frequency chirping of the driving field, which concentrate a large portion \\ of the total energy of the metamaterial within only a few lattice sites.
}

PACS numbers: 05.45.-a, 05.45.Xt, 63.20.Pw

\section{Introduction}

Nonlinear localized excitations in the form of discrete breathers (DBs) can be produced spontaneously in discrete lattices of weakly coupled elements either statistically $[1,2]$ or by a purely deterministic mechanism that relies on the modulational instability (MI) of propagating waves in nonlinear media $[3,4]$. DBs are spatially localized, time-periodic and stable excitations, and they have been observed experimentally in a variety of physical systems [5]. The last decade, the study of metamaterials [6-9], i.e., of artificially structured composites that exhibit electromagnetic properties not available in naturally occuring materials, was really intense. Among them, the magnetic metamaterials (MMs), which exhibit significant magnetic properties up to $\mathrm{THz}$ and optical frequencies $[10,11]$, are typically comprised of split-ring resonators (SRRs) [12]. It was recently demonstrated that DBs can be generated in nonlinear MMs, both in one and two dimensions [13-16]. The simplest SRR is just a highly conducting metallic ring with a slit, where a capacitance is built up. SRR-based MMs may become nonlinear by insertion, for example, of a varactor into the slit of each SRR [17-19]. The nonlinearity offers real-time tunability of the metamaterial parameters by varying the input power [20]. Besides DBs, other types of nonlinear localized excitations, such as magnetic domain walls and envelope solitons, may also appear in those systems $[21,22]$. In the present work we consider a MM comprised of two types of nonlinear SRRs, which are placed alternatingly in a one-dimensional periodic lattice [23]. It has been recently suggested that such a binary MM is well suited for the observation of second harmonic gen-

* corresponding author; e-mail: nl@physics.uoc.gr eration [24]. The binary structure of that MM allows for the spontaneous generation of dissipative DBs which are directly induced by MI, using frequency chirping of the driving field $[4,23]$. The frequency chirping method seems almost ideal for the generation of dissipative DBs in experiments involving nonlinear binary MMs. In the next section we give the basics for the model nonlinear binary MM, while in Sect. 3 we explain how the frequency chirping method is used for the generation of DBs. We finish in Sect. 4 with the conclusions.

\section{Nonlinear binary metamaterial model}

Consider a one-dimensional binary MM comprised of two types of SRRs, say type "a" and type "b", placed alternatingly in a periodic lattice. Each SRR, that is assumed to possess a capacitive nonlinearity, is considered as a nonlinear resistor-inductor-capacitor (RLC) resonator. The SRRs differ in their slit widths, resulting in different linear resonance frequencies $[23,24], \omega_{a}$ and $\omega_{b}$. The resonance frequency mismatch (RFM) is quantified with the parameter $\delta=\omega_{a} / \omega_{b}$, which enters into the dynamic equations for the charges across each SRR slit [23]. The nonlinear MM is irradiated by an alternating electromagnetic field whose polarization has been chosen so that its magnetic component is perpendicular to the SRR planes, while its electric component is perpendicular to their slits. Thus, it is the magnetic component of the field that induces an electromotive force in each SRR. The SRRs are coupled to their nearest neighbors through magnetic dipole-dipole interactions due to their mutual magnetic inductance, $M$, which can be either positive or negative, depending on the relative orientation of the SRRs in the array [14]. If the location of the slits is such that they are relatively far apart, the electric dipole-dipole interactions between SRRs can be 
neglected. Then, the equation for the normalized charge $q_{n}$ accumulated in the capacitor of the $n$-th SRR reads

$$
\begin{aligned}
& \frac{\mathrm{d}^{2}}{\mathrm{~d} \tau^{2}}\left(\lambda q_{n-1}+q_{n}+\lambda q_{n+1}\right)+\gamma \frac{\mathrm{d} q_{n}}{\mathrm{~d} \tau}+\omega_{n}^{2} q_{n}-\chi \omega_{n}^{6} q_{n}^{3} \\
& \quad=\varepsilon_{0} \sin (\Omega \tau),
\end{aligned}
$$

where $\lambda=M / L$ is the magnetic coupling parameter with $L$ being the inductance of the SRR, $\chi$ is the nonlinearity parameter, $\gamma$ is the loss coefficient, $\tau$ is the normalized temporal variable, $\varepsilon_{0}$ and $\Omega$ are the amplitude and frequency, respectively, of the induced electromotive force, and $\omega_{n}^{2}=1 / \delta\left(\omega_{n}^{2}=\delta\right)$ for $n$ an odd (even) integer. The dispersion law for linear waves, which has two branches separated by a gap, is obtained as

$$
\begin{aligned}
& \Omega_{ \pm}^{2} . \\
& =\frac{(\delta+1 / \delta) \pm \cdot \sqrt{(\delta+1 / \delta)^{2}+4\left(1-4 \lambda^{2} \cos ^{2} \kappa\right)}}{2\left(1-4 \lambda^{2} \cos ^{2} \kappa\right)} .
\end{aligned}
$$

In the absence of dissipation and external driving, the corresponding dynamic equations can be obtained from the Hamiltonian $H=\sum H_{n}$, where the discrete energy density $H_{n}$ is given by

$$
\begin{aligned}
& H_{n}=\frac{1}{2} \dot{q}_{n}^{2}+\frac{1}{2} \lambda \dot{q}_{n}\left(\dot{q}_{n-1}+\dot{q}_{n+1}\right) \\
& +\frac{1}{2} \omega_{n}^{2} q_{n}^{2}\left(1-\frac{1}{2} \chi \omega_{n}^{4} q_{n}^{2}\right) .
\end{aligned}
$$

The on-site nonlinear potential $V_{n}$, given by the last term of the earlier equation, is hard (soft) for $\chi<0(\chi>0)$.

\section{Dissipative breather induction by modulational instability}

For a frequency gapped linear spectrum, some of the linear modes become unstable at large amplitude. If the curvature of the dispersion curve in the region of these modes is negative and the on-site potential is hard then, these modes become unstable with respect to formation of localized excitations in the form of DBs in the gap above the upper linear band [4]. We choose the RFM and coupling parameters to be $\delta=2$ and $\lambda=0.05$, respectively, so that the top of the upper band is at frequency $\Omega_{0}=1.42$. Around that frequency, the dispersion Eq. (2) exhibits negative curvature, while for negative nonlinearity parameter $\chi$ the on-site potential is hard. We start driving the system strongly with frequency just below $\Omega_{0}$, in order to produce a large vibrational amplitude of the uniform mode. The frequency is chirped with time and when it approaches $\Omega_{0}$ the instability sets in, leading to spontaneous DB generation. During the chirping phase a number of DBs is generated, and some of them become frequency-locked to the driver and survive until the end of that phase. Then, the driving frequency is well above $\Omega_{0}$, and it is kept constant during the next phase. During the constant frequency phase, the DBs that survived continue to receive energy from the driver and they fall into a stationary state. When the driver is switched off, the DBs die in a short time interval.

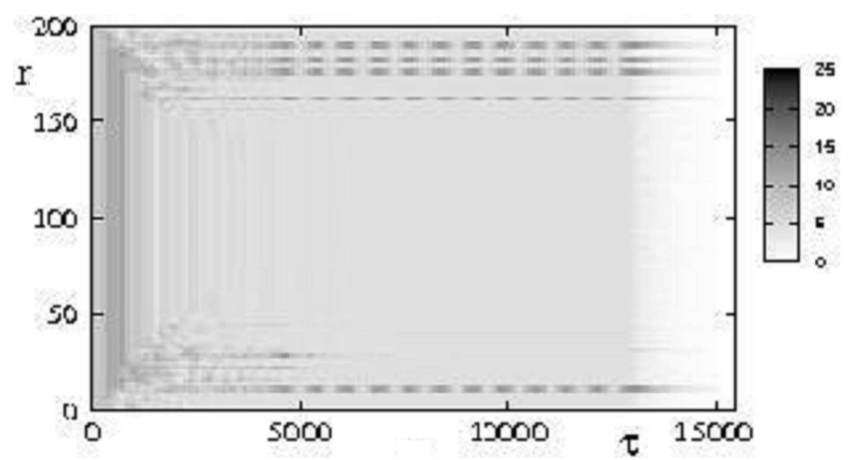

Fig. 1. Gray scale plot of the energy density $H_{n}$ on the $\tau-n$ plane for a binary magnetic metamaterial with $\Omega_{0}=1.42, \chi=-1 / 6, \gamma=0.001, \lambda=0.05, \delta=2$, $N=200$, and $\varepsilon_{0}=3$.

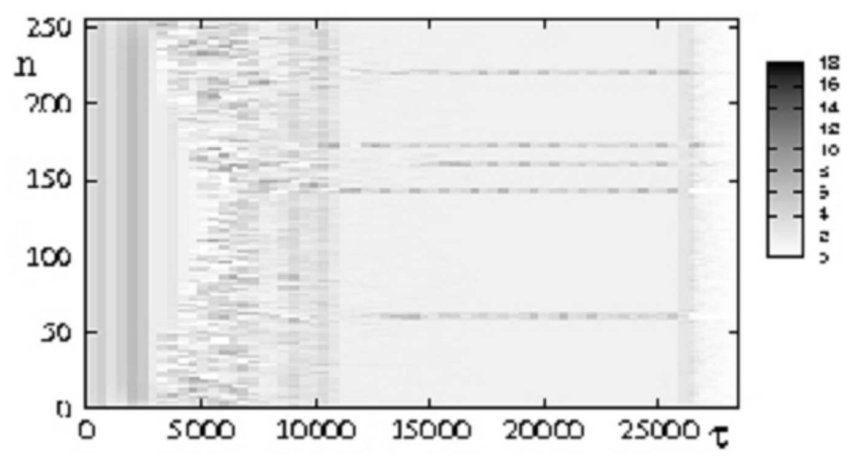

Fig. 2. Gray scale plot of the energy density $H_{n}$ on the $\tau-n$ plane for a binary magnetic metamaterial with $\Omega_{0}=1.42, \chi=-1 / 6, \gamma=0.001, \lambda=0.05, \delta=2$, $N=256$ and $\varepsilon_{0}=2$.

In Fig. 1 we plot in gray scale the energy density $H_{n}$ on the $\tau-n$ plane for a nonlinear binary MM, where darker color corresponds to higher energy. The chirping phase lasts for $\tau_{\mathrm{ch}}=1000 T_{0} \sim 4425$ time units, with $T_{0}=2 \pi / \Omega_{0}$, during which the frequency varies linearly from $\Omega_{\mathrm{I}}=0.997 \Omega_{0}$ to $\Omega_{\mathrm{F}}=1.020 \Omega_{0}$. At the end of that phase, a number of DBs which are locked to the driver and are trapped at particular sites have been formed, indicated in the figure by the dark horizontal lines. Those DBs seem to survive during the whole time interval of the constant frequency phase, which lasts for $\tau_{\text {cf }}=2000 T_{0} \sim 8850$ time units. Eventually, all DBs die out when the driver is switched off. The same quantity is plotted in Fig. 2, where a number of DBs have been apparently generated and can be seen clearly in the figure during the constant frequency phase. In this case, the chirping rate was slower than that used in Fig. 1, i.e., the frequency variation between $\Omega_{\mathrm{I}}$ and $\Omega_{\mathrm{F}}$ occurred during time $\tau_{\mathrm{ch}}=2500 T_{0} \sim 11000$ time units. Except that, the two figures differ in the array size and driving amplitude. The constant frequency phase also lasts longer than before $\left(\tau_{\text {cf }}=3500 T_{0} \sim 15500\right.$ time units $)$. 


\section{Conclusions}

In conclusion, we have applied the frequency chirping method to a driven nonlinear binary SRR-based MM with dissipation, in order to generate dissipative DBs, i.e., discrete breathers that can exist as a result of a power balance between the incident power and the intrinsic power losses. It seems that we can generate DBs in this way for metamaterial parameters that may vary in rather wide intervals. Although DB generation with frequency chirping seems to be rather generic, the number of the DBs which become frequency-locked to the driver and their energy depend crucially on the specific choice of the parameters. Until now, the frequency chirping method for DB generation in dissipative - driven systems have been applied successfully in di-element cantilever arrays [4]. Generally is seems easier to generate DBs in di- or multi-element arrays, probably because they are more easily affected to instabilities. Recently, a three-dimensional localized breather was detected in the diatomic ionic crystal $\mathrm{NaI}[25]$.

\section{References}

[1] G.P. Tsironis, S. Aubry, Phys. Rev. Lett. 77, 5225 (1996).

[2] K.O. Rasmussen, S. Aubry, A.R. Bishop, G.P. Tsironis, Europ. J. Phys. B 15, 169 (2000).

[3] D. Hennig, L. Schimansky-Geier, P. Hanggi, Europhys. Lett. 78, 20002 (2007).

[4] M. Sato, B.E. Hubbard, A.J. Sievers, B. Ilic, D.A. Czaplewski, H.G. Craighead, Phys. Rev. Lett. 90, 044102 (2003).

[5] S. Flach, A.V. Gorbach, Phys. Rep. 467, 1 (2008).

[6] S. Linden, C. Enkrich, G. Dolling, M. W. Klein, J. Zhou, T. Koschny, C.M. Soukoulis, S. Burger, F. Schmidt, M. Wegener, IEEE J. Selec. Top. Quant. Electron. 12, 1097 (2006).

[7] C.M. Soukoulis, S. Linden, M. Wegener, Science 315, 47 (2007).
[8] V.M. Shalaev, Nat. Photonics 1, 41 (2007).

[9] N.M. Litchinitser, V.M. Shalaev, Laser Phys. Lett. 5, 411 (2008).

[10] T.J. Yen, W.J. Padella, N. Fang, D.C. Vier, D.R. Smith, J.B. Pendry, D.N Basov, X. Zhang, Science 303, 1494 (2004).

[11] N. Katsarakis, G. Konstadinis, A. Kostopoulos, R.S. Penciu, T.F. Gundogdu, M. Kafesaki, E.N. Economou, Th. Koschny, C.M. Soukolis, Opt. Lett. 30, 1348 (2005).

[12] M. Kafesaki, Th. Koschny, J. Zhou, N. Katsarakis, I. Tsiapa, E.N. Economou, C.M. Soukoulis, Physica B: Cond. Matt. 394, 148 (2007).

[13] N. Lazarides, M. Eleftheriou, G.P. Tsironis, Phys. Rev. Lett. 97, 157406 (2006).

[14] M. Eleftheriou, N. Lazarides, G.P. Tsironis, Phys. Rev. E 77, 036608 (2008).

[15] N. Lazarides, G.P. Tsironis, Yu. S. Kivshar, Phys. Rev. E 77, 036608(R) (2008).

[16] M. Eleftheriou, N. Lazarides, G.P. Tsironis, Yu.S. Kivshar, Phys. Rev. E 80, 017601 (2009).

[17] I.V. Shadrivov, S.K. Morrison, Yu. S. Kivshar, Opt. Express 14, 9344 (2006).

[18] D.A. Powell, I.V. Shadrivov, Yu. S. Kivshar, M.V. Gorkunov, Appl. Phys. Lett. 91, 144107 (2007).

[19] B. Wang, J. Zhou, T. Koschny, C.M. Soukoulis, Opt. Express 16, 16058 (2008).

[20] I.V. Shadrivov, A.B. Kozyrev, D. van der Weide, Yu.S. Kivshar, Appl. Phys. Lett. 93, 161903 (2008).

[21] I.V. Shadrivov, A.A. Zharov, N.A. Zharova, Yu.S. Kivshar, Photonics Nanostruct. Fundam. Appl. 4, 69 (2006).

[22] I. Kourakis, N. Lazarides, G.P. Tsironis, Phys. Rev. E 75, 067601 (2007).

[23] M.I. Molina, N. Lazarides, G.P. Tsironis, arXiv:0905.4474v1

[24] M.V. Gorkunov, I.V. Shadrivov, Yu.S. Kivshar, Appl. Phys. Lett. 88, 071912 (2006).

[25] M.E. Manley, A.J. Sievers, J.W. Lynn, S.A. Kiselev, N.I. Agladze, Y. Chen, A. Llobet, A. Alatas, Phys. Rev. B 79, 134304 (2009). 